\title{
DIAGNÓSTICO PARA UN CAMBIO: LOS DILEMAS DE LA REGULACIÓN DE LAS AGUAS EN CHILE
}

\author{
DIAGNOSIS FOR A CHANGE: CHILEAN WATER REGULATION \\ DILEMMA'S
}

\section{Ezio Costa Cordella ${ }^{*}, * *$}

\begin{abstract}
RESUMEN: La regulación de las aguas en Chile es un tema candente para el derecho y las políticas públicas. Empujado por una serie de conflictos sociales en torno a este bien, se hace inminente la necesidad de efectuar algunas modificaciones en esta área. Sin embargo, se observa la falta de un diagnóstico efectivo sobre los diversos dilemas que envuelve la regulación de aguas. Este trabajo constituye una revisión de literatura jurídico-política sobre la regulación de aguas que busca clasificar los problemas que sobre ella se han detectado. Con este objetivo es que en una primera parte se analizan las principales caracterísiticas del sistema actual y en seguida se clasifican las problemáticas en 4 "dilemas", de diversas características y que debieran significar esfuerzos distintos para abordar sus soluciones.
\end{abstract}

Palabras clave: Derecho de aguas, regulación de aguas, cambio regulatorio, agua, bien nacional de uso público.

ABSTRACT: Water Regulation in Chile has been a problematic issue for law and public policies. A series of social conflicts regarding this good has led to a scenario where the change is imminent and needed. However, there is a lack of a proper diagnosis of what are the problems that water regulation has to solve. This article reviews the relevant law and public policies literature regarding the subject and creates a list of the detected problems. With this objective in mind, the first part of the article analyze the main features of the current water regulation and in a second part it classifies the problems in four "dilemmas", each one with different features and that should mean different efforts to solve them.

Key words: Water law, water regulation, regulatory change, water policy, public good

\section{INTRODUCCIÓN}

Un tema que aparece de manera intermitente en la agenda pública es la regulación y el derecho de aguas, lo que es natural a la luz de que el agua dulce es un recurso escaso e indispensable para la vida, la protección ambiental y la producción económica. Cuestiones como el cambio climático, la creciente presión sobre el recurso, un aumento en los conflictos y las fallas regulatorias del sistema chileno, han puesto el asunto en un punto crítico en lo que respecta la relación de las personas en torno al agua. Entender la crisis del agua re-

\footnotetext{
* Abogado, Universidad de Chile. Msc en Regulación, London School of Economics and Political Science, Investigador del Centro de Regulación y Competencia, Facultad de Derecho, Universidad de Chile. Dirección Postal: Pío Nono N¹, cuarto piso, Providencia, Santiago. Dirección electrónica: ecosta@derecho.uchile.cl

** Agradezco la ayuda del equipo de RegCom y en especial el trabajo de Natalia Acevedo Alvear y los comentarios de Nicolás Rojas Covarrubias.
} 
quiere de una comprensión multisectorial de cuáles pueden ser las causas y los factores que contribuyen en el asunto. Así entonces, diversas iniciativas se vienen llevando a cabo desde diversas ciencias y disciplinas.

Desde la Regulación, un primer aporte es intentar entender cómo se configuran los problemas que enfrenta el sistema normativo del agua. En efecto, en la discusión pública y académica hay diversos asuntos que se tratan e interconectan, siendo que no todos ellos son abordados con la profundidad necesaria. En especial, es observable que en diversas ocasiones se construyen vínculos automáticos entre un tipo de problemas y otros, lo que podría ser perjudicial a la hora de intentar soluciones, por bien intencionadas que estas sean.

El presente trabajo entonces, consiste en un diagnóstico de los problemas que enfrenta la regulación de aguas en este momento. No se pretende abordar cada uno de los puntos normativos específicos en que un cambio podría ser positivo, ni tampoco cada una de las críticas que se le hacen a la actual normativa, sino que clasificar los problemas comunes que se reconocen. En este sentido, la tipología propuesta intenta ser un aporte a la discusión pública y a la investigación académica sobre el tema, generando una base de discusión común que permita soluciones racionales y efectivas.

Este trabajo hace, en primer lugar, una brevísima descripción de las principales características de la regulación actual de las aguas en Chile, con el solo objeto de contextualizar. En seguida y partiendo del paradigma del cambio regulatorio, construye una tipología sobre los dilemas del agua, la cual pretende abarcar las cuestiones principales sobre las que se basan las críticas al sistema actual, generando parcelas sobre las cuales se pueda accionar de manera diferenciada con instrumentos de política pública.

Se reconocen cuatro dilemas, cada uno con sus determinadas características. En primer lugar, se trata el dilema ideológico, un asunto que arrastra al menos varios cientos de años y que genera una tensión permanente entre los actores políticos alrededor de los asuntos de aguas. En seguida, se trata la escasez material del recurso y cómo ella genera tensiones a cualquier sistema regulatorio. En tercer lugar se especifican los problemas de acceso y disponibilidad, ligados a cuestiones valóricas y normativas, como el derecho humano al agua, la disponibilidad jurídica del recurso y la protección del medio ambiente. Por último, se comenta el dilema de institucionalidad, entendiendo por esto las dificultades de la organización administrativa en relación con este asunto.

\section{REGULACIÓN DE LAS AGUAS, PRINCIPALES CARACTERÍSTICAS}

En esta sección se analizarán las tres principales características del sistema regulatorio del agua. La revisión se realiza a objeto de ilustrar cómo dichas características influyen en el diseño institucional-organizativo del agua y para fijar ciertos conceptos que serán tratados en los acápites posteriores. No se pretende un análisis exhaustivo sobre estas características, sino iluminar ciertos aspectos relevantes para el objetivo principal de la investigación.

\section{A. El mercado del aGua}

La primera y principal característica de la regulación actual de las aguas es el hecho de que los derechos de aguas están concebidos como derechos reales, apropiables y transa- 
bles en el mercado. El sistema -como tantos otros en Chile- es el fruto de una construcción neoliberalista, donde la propiedad privada era vista como la única y mejor manera de lograr estabilidad, eficiencia y justicia. Esta idea fue aplicada de manera absoluta, aprovechando la contingencia política chilena. Como señala Celume, "La época de promulgación

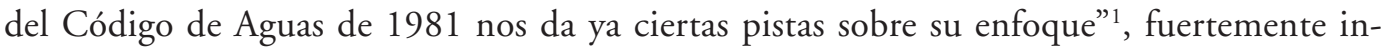
fluido en términos económicos por el neoliberalismo.

Por lo mismo no resulta tan curioso que Vergara haga variadas referencias a la dicotomía Estado-Mercado cuando explica lo que él llama el "Neomoderno Derecho de Aguas", pues al explicarlo desde esa lógica lo sitúa precisamente en el momento histórico en que fue concebido y asimismo lo dota de cierto contexto, necesario para intentar comprenderlo ${ }^{2}$. Para el mismo autor, el Mercado de Aguas es una de las características principales del sistema regulatorio chileno, cuestión que en cualquier caso es ampliamente reconocida ${ }^{3}$.

Bauer también reconoce la creación del mercado de aguas como uno de los dos principales fines de la dictación del Código de Aguas, conjuntamente con fortalecer la seguridad jurídica de los derechos de aguas. En este sentido, reconoce una tensión en la discusión interna del régimen, entre los partidarios del neoliberalismo que pretendían una mayor profundidad del mercado y movilidad de los derechos de aguas, y los agricultores y conservadores que si bien apoyaban la creación de un mercado, abogaban por mayor seguridad jurídica y, entre otras cosas, que no se cobrara un tributo especial por las aguas separadas de la tierra, cuestión que prevaleción ${ }^{4}$.

Las características de este mercado son bien conocidas. La Dirección General de Aguas (en adelante DGA) entrega a los particulares que lo solicitan un derecho de consumo o de uso de aguas, con determinadas características. Ese derecho, calificado como real por la ley, es perpetuo y transferible, creándose entonces un mercado que si bien tiene un número importante de falencias, cumple con su rol principal como distribuidor de la escasez. Además, en un aspecto que es calificado por Vergara como principio del derecho de aguas actual, se asegura la libre transferibilidad de los derechos ${ }^{5}$.

La existencia del mercado como arreglo institucional en esta área no está exenta de polémica y de hecho es probablemente un punto central en la discusión. Desde el punto de vista ético e ideológico, se ha reclamado entre otras cosas que la existencia de este mercado atenta contra el derecho al medio ambiente sano, impide la realización del derecho humano al agua, es el producto de una política ilegítima y entra en claro conflicto con la calidad de bien nacional de uso público que el agua detentaría por su propia naturaleza y de acuerdo a lo establecido en nuestro derecho ${ }^{6}$.

Desde lo práctico, los reproches apuntan por una parte al hecho de que un bien como el agua podría ser administrado mediante otros arreglos institucionales que benefi-

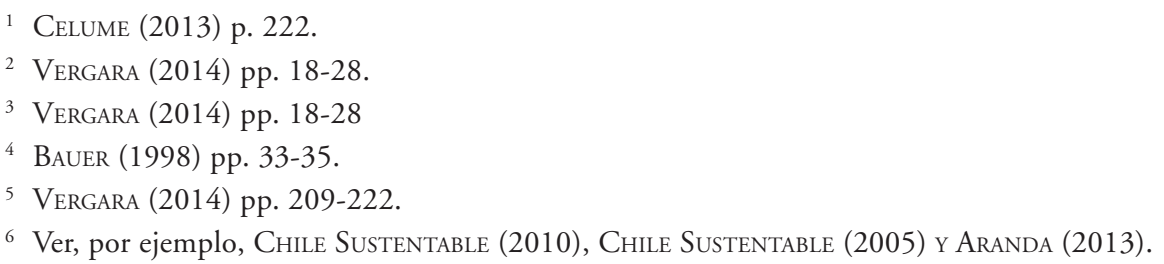


ciarían tanto a los usuarios como a la conservación del recurso. En esta línea es posible observar las críticas que apuntan a la no caducidad de los derechos de aguas, al hecho de que ellos no se encuentren condicionados a ciertos usos y al que la entrega y mantención de los derechos sea a título gratuito.

En cualquier caso, el sistema regulatorio actual del agua descansa principalmente en la existencia de este mercado y las posibilidades teóricas que la existencia de este arreglo implican, siendo así que las demás características que se exploran responden igualmente a los supuestos de la existencia de un mercado funcional.

\section{a. La gestión por parte de los usuarios (o autogestión)}

Una segunda característica fundamental en nuestro sistema de aguas es que son los propios usuarios del agua quienes están encargados de cumplir un rol en la gestión y distribución del recurso, para lo cual deben agruparse en las organizaciones de usuarios de agua (OUA), siendo la más importante la Junta de Vigilancia, que supuestamente debiera agrupar a todos los usuarios de una misma cuenca ${ }^{7}$.

La autogestión tiene una importancia crucial en el manejo de un recurso común como es el agua y así se ha reconocido en diferentes realidades. A este respecto, es muy importante el trabajo de Ostrom, quien estudiando el manejo de recursos de uso común -entre ellos el agua- ha definido o reconocido la existencia de ciertos principios comunes a los modelos que han sido exitosos en la materia. Estos principios, que sirven para determinar las regulaciones con las que los usuarios de un recurso común se relacionan, son denominados por la autora como principios de diseño y entre ellos se reconocen al menos tres que están en directa relación con la autogestión: (i) Los arreglos de elección colectiva, (ii) el reconocimiento mínimo de derechos de organización, y (iii) los mecanismos para la resolución de conflictos ${ }^{8}$.

El primer principio se basa en la idea de que la mayor parte de las personas que son afectadas por la administración del recurso deben tener la posibilidad de participar en la decisión relativa a las reglas con las cuales se lleva a cabo, así como tener la posibilidad de promover y participar en la modificación en las mismas reglas. La utilidad de este principio se encuentra en que las instancias locales son más aptas para adecuar las reglas a la realidad particular que enfrentan?.

El segundo principio es el reconocimiento de mínimos derechos de organización, que supone la posibilidad de los usuarios de fijar sus propias instituciones sin la intervención de autoridades gubernamentales externas ${ }^{10}$. Por último, encontramos los mecanismos de solución de conflictos, los que debieran ser en instancias locales que resuelvan los problemas a bajo costo.

\footnotetext{
7 Se dice "supuestamente" por cuanto a pesar de la existencia del principio de Unidad de Corriente, se ha aceptado la constitución de varias Juntas de Vigilancia en un mismo cauce cuando se dan ciertas condiciones, lo que ha provocado que haya una especie de competencia por el recurso entre distintas secciones de un mismo río.

8 Ostrom (2012).

9 Ostrom (2012) pp. 171-172.

10 Ostrom (2012) pp. 182-183.
} 
En la realidad regulatoria chilena se observan al menos dos temas relevantes que no son abordados adecuadamente, a pesar de reconocer la importancia de la autogestión y promoverla. El primero es el hecho de que no todos los afectados por el manejo del recurso común pueden participar de las decisiones que se toman sobre él ni resolver los conflictos que se generan en torno a él. En efecto, las Juntas de Vigilancia integran solamente a las personas que tienen actualmente derechos de agua en una determinada cuenca (o sección), sin que se tome en consideración a los demás individuos que pudieren verse afectados por las decisiones a pesar de no ser titulares de derechos ${ }^{11}$. En este mismo sentido, las reglas referentes al poder político dentro de las organizaciones tampoco es igualitaria, sino que en proporción a los derechos poseídos por cada usuario, provocando una posición políticamente privilegiada para quienes poseen más derechos de aguas y por lo tanto, concentración de poder.

Por otra parte, existe cierta rigidez en las reglas que las Organizaciones de Usuarios de Aguas (en adelante, OUA) deben aplicar, siendo el caso que aun cuando estas tienen cierta libertad para modificar el reparto, fijar turnos y otras cuestiones meramente administrativas, no tienen dentro de sus herramientas la posibilidad de modificar instituciones de nivel superior, siendo entonces imposible lograr acuerdos sociales significativos.

Para Celume, este sistema está directamente relacionado con la limitación de las funciones de las autoridades gubernamentales, con miras a descentralizar la gestión de los recursos hídricos a través de la privatización de dicha función ${ }^{12}$. Esta visión precisamente hace el puente necesario entre las dos primeras características fundamentales que se han analizado, y la tercera. Mientras se fortalece el mercado y la gestión por parte de los usuarios, la balanza se carga en contra de las funciones de la Administración.

\section{b. El rol de la Administración}

El rol de la Administración en la regulación de las aguas es bastante limitado en el sistema creado por el Código de Aguas de 1981, sin perjuicio de que existen muchos organismos con distintos poderes relativos al tema. La DGA es el organismo más representativo de la institucionalidad de aguas, pero no tiene competencias exclusivas, sino que convive con 43 organismos que se reparten las 102 funciones relativas al agua ${ }^{13}$, incluyendo aquellas funciones públicas atribuidas a los organismos privados, de extrema relevancia en la materia, en especial las Juntas de Vigilancia ${ }^{14}$.

Los órganos de la Administración tienen una serie de potestades relacionadas con los recursos hídricos, pero no tienen un papel relevante en la gestión del agua. De hecho, a pesar de la gran cantidad de organismos que intervienen en este asunto, los autores en general reconocen que su influencia es limitada. En este sentido, considera Riquelme, por ejemplo,

11 Se incluye en ese sentido a todos los habitantes de una cuenca, en cuanto las decisiones de las organizaciones de usuarios podrían impactar el ciclo hidrológico y con ello a la totalidad de las actividades que se realizan en esa cuenca.

12 Celume (2013) p. 233.

13 Es necesario considerar, sin embargo, que dentro de esos 43 organismos están incluidos todos los poderes del Estado e incluso los organismos privados como las OUA.

14 Banco Mundial (2013a). 
que "la desvinculación de la Autoridad respecto del acto que constituye los derechos de aprovechamiento nos parece, de sumo, peligroso" ${ }^{15}$, agregando que el resguardo del interés público en un mercado como el de las aguas requeriría de una autoridad con otras características y mayores facultades regulatorias.

Los roles de la administración varían de acuerdo al organismo que se observe. En el caso de la DGA están principalmente circunscritos a la asignación inicial del recurso y la mantención de un sistema de información sobre el mismo; el catastro público de aguas, mientras que el grueso de las facultades de gestión y administración, e incluso la resolución de conflictos están fuera de su ámbito de competencia y entregadas a las OUA o los Tribunales.

La crítica a la escasa injerencia de la Administración alcanza incluso a su poca participación en los arreglos fundamentales para hacer que el mercado del agua funcione. En este sentido Herne y Donoso consideran que "apoyado en la política de libre mercado, el gobierno de Chile no tiene un objetivo particular en mejorar el mercado de aguas, más allá de proveer un marco de acción para el desarrollo de ese mercado" ${ }^{16}$. Como estima Bauer, el rol de la DGA es un rol de carácter subsidiario con poca autoridad para intervenir en conflictos por el uso del agua ${ }^{17}$.

La más cruda visión es la de Vergara, ve la "anomia administrativa" como una de las crisis del agua. Para el autor, la DGA tiene solo tres fines: hacer un inventario del recurso, catastrar títulos de particulares y ayudar a la regularización de los derechos de aguas, cuestiones respecto de las cuales "se encuentra casi absolutamente impedido de realizar de manera eficiente, adecuada o mínimimamente satisfactoria"18.

En suma, la participación de la administración en la gestión de las aguas se encuentra disminuida en la regulación actual, siendo que además de ello está atomizada y es poco clara, de tal manera que difícilmente puede tener una influencia real sobre la gestión del recurso. Esta configuración obedece a una visión política y económica preponderante al momento de dictarse el Código de Aguas, pero parece claro que la configuración de las potestades estatales no sería suficiente ni siquiera para satisfacer las necesidades propias de la creación de un mercado de aguas.

\section{B. Los Dilemas DE LA REgUlaCión DE AGUAS}

\section{i. El dilema ideológico}

Para entender la existencia de un problema ideológico hay que hacer un análisis de diversos textos que lo tratan de manera más o menos expresa, dando cuenta de su existencia. Uno que lo hace de manera muy expresa es Dourojeanni y Jouravlev en su artículo "El Código de Aguas de Chile, entre la ideología y la realidad", donde señalan que el sistema de aguas en Chile está "fuertemente desbalanceado en favor de la protección de derechos de propiedad sobre la concesión de uso del agua -que en Chile, por la forma de otorgamiento que se hace

\footnotetext{
15 Riquelme (2014) p. 302.

16 Heane y Donoso (2014) p. 118.

17 Bauer (1998) p. 90.

18 Vergara, (2014) p. 15.
} 
de dicha concesión se convierte en la práctica en un derecho de propiedad del agua- y en perjuicio de las normas regulatorias" ${ }^{19}$ y critican puntualmente que los derechos se entreguen de manera incondicionada, se fomente la especulación y acaparamiento, se afecte el desarrollo regional, se promueva la transferencia de un bien público importante a particulares y de manera gratuita, permite ejercer poder de mercado, ha dado lugar a la aparición de monopolios y ha producido externalidades ${ }^{20}$.

Curiosamente además, entre los desequilibrios fundamentales que los autores mencionan, está especialmente reconocido que el sistema de derechos de agua "...(ii) impide introducir modificaciones en el Código de Aguas y tiene como resultado que la oposición a su modificación se ha planteado en términos muy ideológicos y generales" ${ }^{21}$. Vale decir, que la existencia de un problema ideológico en torno al agua sería un efecto de la configuración institucional del propio sistema.

En el mismo sentido, más recientemente Riquelme ha señalado a propósito de la calidad de bien nacional de uso público del agua que "a nuestro juicio, lo que determina el mayor o menor nivel de protección de los recursos hídricos y su utilización destinada al cumplimiento del bien común, no es su consagración constitucional como (dominio público) bienes nacionales de uso público, sino el modelo de desarrollo que impera en una determinada sociedad”22. Y agrega que "...la incorporación del dominio público hidráulico debiera apuntar a la regulación del mercado de los derechos de aprovechamiento de las aguas, modificándose para ello la redacción del artículo $19 \mathrm{~N}^{\circ} 23$ y $\mathrm{N}^{\circ} 24$, como sustento elemental de nuestro sistema económico neoliberal"23.

Ambas afirmaciones se encuentran en las conclusiones de su artículo y como se observa, la autora hace una importante declaración de principios en torno al tema tratado pues considera que finalmente cualquier modificación de la regulación de las aguas debiera pasar por un cambio del "modelo de desarrollo" o el "sistema económico neoliberal", ambos componentes estructurales que escapan de la mera modificación normativa específica y se ubican en el plano de las ideologías.

Otro ejemplo en este sentido lo encontramos en Aranda. En su artículo sobre la regulación de derechos de aprovechamiento, el autor señala entre los motivos que justificarían intervenir en el actual sistema regulatorio el hecho de "los procesos de privatización y desregulación que han dominado, queramos o no, los últimos treinta años en Chile, han vuelto a colocar sobre el tapete la vieja discusión de qué le corresponde realizar al Estado y hasta donde" ${ }^{24}$, para luego señalar que el Código de Aguas estaría sustentado en un para-

19 Dourojeanni y Jouravlev, (1999), p. 10. Cuando los autores se refieren a normas regulatorias lo hacen en contraposición a las normas estructurales y definiéndolas como aquellas que aseguran el uso y control del agua en virtud de los objetivos económicos, ambientales y sociales.

20 Dourojeanni y Jouravlev (1999) p. 10.

21 Dourojeanni y Jouravlev (1999) p. 11.

22 Riquelme (2014) p. 303.

23 Riquelme (2014) p. 303.

24 Aranda (2013) p. 107. 
digma económico tradicional que le impide considerar las externalidades en su manejo por los privados ${ }^{25}$.

Por último, Vergara a lo largo de su libro sobre la crisis institucional del agua hace variadas alusiones al problema ideológico. Así, por ejemplo, se refiere a que el Código de Aguas "se dicta en medio (al inicio mejor dicho) de una de las más profundas reformas que han operado en nuestro país en las últimas décadas. La redefinición de las relaciones entre el Estado y la sociedad, estableciendo nuevos fundamentos y alterando así las bases de la economía del país" 26 , añadiendo que esta transformación es "del estatismo al libre mercado" y calificando las críticas ideológicas como "pompas de jabón un poco anacrónicas que hoy escuchamos, que nos llaman a reestatizar el agua, a renacionalizar el agua [las cuales], se difuminarán..." 27.

Como es posible observar, los autores se debaten en parte debido a estas preferencias ideológicas, siendo que existe una tensión natural entre quienes creen que el modelo actual no se corresponde con los valores fundamentales de una sociedad, y otros que sí creen que el sistema actual tiene los elementos necesarios para concretar dichos valores fundamentales. A mayor abundamiento, buena parte de la discusión que se produce a nivel de opinión pública está basada principalmente en este dilema ${ }^{28}$ y de acuerdo a los estudios de opinión, un $82,8 \%$ de la población preferiría que el agua fuera de propiedad exclusiva del Estado, superando ampliamente a otros recursos naturales como la pesca y los recursos forestales, e incluso situándose por sobre el cobre ${ }^{29}$.

\section{ii. El dilema material}

El problema material está circunscrito a la escasez física del agua. Esta escasez normalmente se vincula con la falta de disponibilidad material y natural (la escasez y disponibilidad jurídica se analiza con el dilema de acceso) y la sobreexplotación, problema que está muy relacionado con la geografía del país y tiene una distribución irregular, pues mientras en algunas zonas de Chile hay abundantes cantidades de agua, en otras la realidad es la contraria.

Así por ejemplo, de acuerdo a un informe de mayo de 2014, la disponibilidad de agua estaría en una situación de escasez (en los términos del programa de Naciones Unidas para los Recursos Hídricos) desde la Región Metropolitana hacia el norte, cuestión que

\footnotetext{
25 ARANDa (2013) p. 115.

26 Vergara (2014) p. 19.

27 Vergara (2014) p. 23.

28 A modo de ejemplo se pueden mencionar el Editorial del diario La Tercera, de fecha 31 de agosto de 2014 que utiliza con el título "Cambio radical en materia de aguas", asegura que "el cambio desde un esquema que reconoce derechos de uso a otro donde el Estado pretenda la propiedad de las aguas afectará gravemente al país". Asimismo, el Editorial del diario El Mercurio de fecha 9 de julio de 2014 titulada "¿Rango constitucional para el agua?" que señala que el debate del asunto "retrotrae a la vieja discusión respecto de si el derecho de propiedad y el mercado, que atomizan las decisiones de una multitud de agentes con intereses contrapuestos, asignan de mejor manera los recursos, o si ello debe provenir de la centralizada e iluminada mirada de la burocracia gubernamental de turno". En el sentido contrario por ejemplo, el Obispo Luis Infanti en Columna Publicada en El Dinamo con fecha 3 de octubre de 2014 y titulada “¿Agua cómo estás?” señala que "Esta vergonzosa entrega (del agua a privados) constitucional, avalada por la Ley de Aguas de 1981, se revela cada día como una ley inmoral, antiética, antisocial e inhumana..."

29 Universidad de Santiago de Chile (2015).
} 
se vería agravada por el uso del agua existente para procesos mineros. El informe vincula además la existencia de conflictos en las comunidades y entre estas y las empresas mineras y agrícolas como un tema vinculado a esa falta de disponibilidad. Asimismo, para el resto del país estima que la disponibilidad material es favorable, en el sentido de que debiera ser suficiente para satisfacer la demanda y que no se encuentra en escasez de acuerdo a los parámetros internacionalmente reconocidos ${ }^{30}$.

En el mismo sentido, un Informe de la Asociación de Empresas de Servicios Sanitarios A.G. sobre la "gestión de la sequía 2014", señala "ya nadie discute que la escasez hídrica llegó para quedarse y no corresponde a una situación coyuntural, sino que estructural” y precisando que dicha escasez afecta al territorio comprendido entre las regiones de Atacama y Biobío ${ }^{31}$.

Este reconocimiento de la escasez como cuestión estructural es muy relevante, pues se prevé que vaya acrecentándose en el futuro en lugar de alivianarse. En particular, el Banco Mundial señala sobre el asunto que "[e]n el futuro la escasez de agua ya restrictiva en las partes norte y central del país, se acentuará debido a los nuevos requerimientos"32. Este mismo organismo conviene que las causas de la escasez son de dos tipos: las primeras tienen que ver con la alta demanda que supone el modelo de desarrollo, en relación con el de la agricultura de exportación, la minería y la generación de electricidad, cuyo incremento supone un aumento en la demanda por el agua, necesaria para todas estas actividades. Una segunda causa sería el cambio climático ${ }^{33}$.

En consecuencia, el manejo de la escasez hídrica por parte de los organismos administrativos que participen de la gestión del recurso se hace crítico. La necesidad, además, pasa no solo por encontrar la manera de enfrentar un problema actual, sino en gestar las instituciones que otorguen la flexibilidad necesaria para que las soluciones puedan implementarse en escenarios cambiantes y cada vez más graves.

En este sentido, resulta interesante la propuesta del Informe de Conflictos por el Agua en Chile, el cual señala que "la desigualdad de recursos hídricos en las diversas regiones del país (...) Hacen necesario y urgente elaborar y aplicar herramientas de planificación... Esta planificación debería incorporar las especificidades de los ecosistemas de cada una de las regiones, estableciendo prioridades y estrategias regionales para la conservación y sus sustentable de las cuencas" 34 .

Enfrentar un problema material de estas características y magnitud requiere de una visión integrada entre los actores. Hoy, las OUA tienen entregada buena parte del poder para solucionar los conflictos entre los usuarios que devengan de la escasez, pero no ellas ni la administración tienen herramientas que permitan gestionar esta escasez con los demás actores relevantes en una cuenca.

\footnotetext{
30 Iniciativa Agua que has de beber (2014).

31 Asociación de Empresas de Servicios Sanitarios AG (2013)

32 Banco Mundial (2011).

33 Banco Mundial (2011).

34 Chile Sustentable (2010) p. 47.
} 
En este sentido se ha pronunciado también Bauer, quien reconoce que la creciente escasez produce conflictos que no pueden ser resueltos de manera privada, siendo necesario fortalecer la gobernanza del agua a través de políticas e instituciones ${ }^{35}$.

\section{iii. El dilema de acceso y disponibilidad}

El problema de acceso tiene tres variables fundamentales: El derecho humano al agua, la disponibilidad jurídica del agua y la protección del patrimonio ambiental. Lo especial de esta categoría es que ella está cruzada por el problema material pero no está determinada por él. Si bien es cierto que a menor disponibilidad material mayores serán los problemas de acceso, no es menos cierto que los tres problemas descritos en esta categoría están determinados por cuestiones normativas que son independientes del problema material. Así por ejemplo, la protección del patrimonio ambiental puede estar perfectamente garantizada normativamente y el hecho de que exista escasez del recurso no le significará un deterioro, a pesar de que en la realidad el cumplimiento de la garantía se vea complicado y se produzcan efectivamente situaciones de deterioro al medio ambiente.

En lo que se refiere al derecho humano al agua, este derecho no está reconocido expresamente en nuestra legislación, pero sí en ciertos instrumentos internacionales. De particular importancia es la Resolución 69/292 de la Asamblea General de la ONU que reconoce "el derecho humano al agua potable y el saneamiento es un derecho humano esencial"36, aunque no es el único instrumento que lo reconoce, pues se encuentra además en otros instrumentos como la Convención ONU sobre la eliminación de toda forma de discriminación contra la mujer y la Convención de Derechos del Niño ${ }^{37}$.

No es fácil, sin embargo, hallar el contenido de este derecho. En un esfuerzo en ese sentido, García ha señalado que el derecho podría ser conceptualizable dentro de los derechos culturales, económicos y sociales, pues demanda una prestación por parte del Estado. Además, en la búsqueda de su posición jurídica, el derecho al agua podría también vincularse y comprenderse a través del derecho a un nivel de vida adecuado y por lo tanto posicionado conjuntamente como el derecho al vestido, la vivienda y la alimentación, o como complementario de este último ${ }^{38}$.

A pesar de que el gran problema que enfrenta el derecho es la falta de reconocimiento expreso en la normativa, su contenido mínimo estaría dado por las obligaciones que de él emanan para los Estados, y que se contienen en las Observación General n¹5 del Comité de Derechos Económicos Sociales y Culturales de Naciones Unidas. Señala García que este derecho contendría garantías de (a) acceso a cantidad mínima para uso personal, doméstico y de producción de alimentos, (b) acceso a agua en base no discriminatoria, (c) acceso físico a instalaciones a distancia prudente, (d) servicios salubres y regulares, (e) seguridad personal en el acceso a agua, (f) distribución equitativa de instalaciones y servi-

\footnotetext{
5 BAUER (2014) pp. 647 y 670.

36 ONU (2010).

37 TAPIA (2014) p. 312.

38 García (2008) pp. 187-194.
} 
cios, (g) planes nacionales de agua transparentes y participativos, y (h) calidad de agua con estándares mínimos ${ }^{39}$.

En el contexto doctrinario nacional, autores como Ochoa y Tapia han analizado el particular, teniendo conclusiones similares en el sentido de reconocer la existencia del derecho humano al agua y su aplicación en el derecho chileno, la que, sin embargo, se ve enfrentada a la concepción del agua como bien económico y termina estando a la deriva y sin ninguna concreción legal ${ }^{40-41}$. En la jurisprudencia, un caso relevante ha sido el resuelto por el Tribunal Constitucional en la causa rol 2039-11, a propósito de una acción de inaplicabilidad por inconstitucionalidad de la norma que permite a las empresas sanitarias el corte del suministro en casos de no pago, cuestión que se cuestionaba como atentatoria contra el derecho a la vida. El Tribunal rechazó la acción, pero el voto de minoría de Gonzalo García Pino quien está por reconocer el derecho de acceso al agua como parte integrante del derecho a la vida, señala: "3. Que sin perjuicio de lo indicado, es preciso señalar que la actividad de la empresa no puede, en caso alguno, entorpecer el acceso al agua potable pues constituye un bien necesario para el desarrollo y existencia del derecho a la vida, constitucionalmente consagrado en el artículo 19, numeral 1º, de la Carta Fundamental”.

Por último, en una interesante tesis, Espinoza analiza cómo la obligación del Estado relacionada al acceso y consumo de agua potable se encuentra presente en normas desde la fundación de las ciudades hasta nuestros días, independientemente del hecho de que las sanitarias se hayan privatizado durante los años 1980. En efecto, la autora cita normas desde el año 1578 en Santiago y termina por vincular el derecho de acceso al agua con el derecho a la vida y el derecho a la salud en la Constitución de 1980. Especialmente interesante las instituciones que cita como garantes del derecho de acceso al agua en zonas urbanas en el derecho actual, las que para ella serían (i) la Ley General de Urbanismo y Construcciones y en especial la obligación de urbanización del artículo $105^{\circ}$ en relación con los artículos 134 y 136 y (ii) La Ley General de Servicios Sanitarios, en especial la interacción que produciría el mercado regulado que llevaría a la expansión de las áreas de cobertura de las sanitarias y la obligación de las empresas sanitarias de prestar servicios a quien se los solicite ${ }^{42}$.

Adicionalmente, la Ley No 18.778 contiene un subsidio para las personas en situaciones económicas vulnerables en relación con el pago de los servicios de agua potable y alcantarillado, que alcanza hasta un 50\% del valor de los primeros $15 \mathrm{~m}$ cúbicos de consumo. Esta herramienta es probablemente una de las más potentes manifestaciones de aseguramiento del acceso al agua, con una prestación directa del Estado. Pero, sin embargo, todo lo expresado respecto de las aguas potables y de las empresas sanitarias alcanza solo a un porcentaje de la población, que es el que se ubica en los sectores urbanos. Si bien es cierto que es la mayoría de la población, no es menos cierto que así como este, la mayoría de los problemas que tienen implicancias en la vida humana son asumidos por las personas que habitan sectores rurales, y en especial por aquellas con menores posibilidades económicas.

\footnotetext{
39 García (2008) p. 195.

40 OCHOA (2011) pp. 213-226.

41 TAPIA (2014).

42 Espinoza (2012).
} 
El problema del acceso al agua en sectores rurales ha sido enfrentado por la política pública principalmente con el establecimiento de los sistemas de Agua Potable Rural (APR) y sin perjuicio de la posibilidad de proveerse de agua mediante un sistema privado y del derecho a extraer aguas subterráneas para usos domiciliarios del artículo $56^{\circ}$ del Código de Aguas.

Sobre la disponibilidad jurídica, el problema estaría dado en la imposibilidad de nuevos usuarios para solicitar aguas cuando las cuencas están agotadas, o de solo pedir derechos eventuales, que en muchos casos nunca llegarán en realidad. Pero aún más, el problema de la disponibilidad jurídica también está relacionado con la sobreexplotación jurídica. En este sentido, es una realidad conocida en nuestro país que las cuencas en general se encuentran sobreotorgadas, de tal manera que hay muchos más derechos de aguas constituidos que el agua que efectivamente está disponible en las cuencas. A este problema se suma el hecho de que en virtud de los problemas existentes con el Catastro Público de Aguas $^{43}$ y la existencia de derechos reconocidos pero no regularizados ni perfeccionados, en general la demanda efectiva de las cuencas no es conocida por la autoridad. A este problema se refieren por ejemplo Prieto y Leyton, dejando constancia del problema que supone para los proyectos mineros el hecho de que a pesar de que cuenten con ciertos caudales de agua teóricos que constan en sus títulos de derechos de aguas, finalmente será la autoridad ambiental la que observando la realidad de las cuencas les otorgará el permiso para utilizar solo un porcentaje de dichos derechos ${ }^{44}$.

Finalmente, nos encontramos con el problema de la protección del patrimonio ambiental. Así como el agua es escasa para el consumo humano, también lo es para abastecer a la naturaleza que depende de ella. De manera evidente, esto afecta la biodiversidad, pues flora y fauna dependen de la disponibilidad de agua para vivir, pero además tiene otros efectos, como la disminución del arrastre de sedimentos, necesarios para la fertilidad de los suelos y para la vida marina, entre otros. Por último, y de mayor relevancia, la falta de disponibilidad de agua puede impactar sobre sí misma, disminuyendo la provisión de agua en una cuenca. En este sentido Little y Lara hacen mención de cómo la cubierta vegetal de las cuencas es uno de los principales factores que determinan la provisión de agua de la misma, siendo en particular el bosque nativo el que mejores rendimientos daría a la cuenca ${ }^{45}$.

La protección del patrimonio ambiental cobra relevancia desde distintos puntos de vista. Desde el meramente legal, porque a ello obliga la Constitución en el inciso segundo del artículo $19 \mathrm{~N}^{\circ}$ 8. Desde un punto de vista económico porque la naturaleza presta servicios ecosistémicos que son difíciles o imposibles de reemplazar y de los que depende no solo la vida humana, sino cualquier actividad económica. Desde un punto de vista ético, por una parte porque el medio ambiente en sí mismo representaría un valor fundamental, y por otra por el concepto de justicia intergeneracional y por lo tanto la obligación de entregar el planeta en condiciones de ser disfrutado por las generaciones que vendrán.

${ }^{43}$ En este sentido los informes del Centro de Regulación y Competencia (RegCom) de la Facultad de Derecho de la Universidad de Chile: "Diagnóstico de las facultades legales y reglamentarias en torno al Catastro Público de Aguas y Propuesta de Nuevo Reglamento para el catastro público de aguas". Ambos disponibles en http:// regcom.cl/documentos-papers-e-informes/

${ }^{44}$ Prieto y Leyton (2010) pp. 231-245.

${ }^{45}$ Little y Lara (2010) pp. 171-178. 
En la legislación de aguas el componente ambiental cobró relevancia recién en 2005 con la creación del "caudal ecológico", cuestión que sin embargo no ha tenido el impacto que se pretendía, recibiendo fuertes críticas. Uno de ellos es Valenzuela, quien señala que "su aporte [del caudal ecológico] se restringe solo a aquellos cauces donde aún hay disponibilidad para constituir nuevos DAA, lo que deriva en un desbalance en el cuidado ambiental entre el norte-centro y sur de Chile"46-47.

Por último, y como reflexiona Boettiger, a pesar de que existen normas destinadas a proteger las variables ambientales del agua, la DGA no tiene facultades generales para la protección ambiental ${ }^{48}$. Peor aún, no se observa que dicha función esté entregada a ningún otro organismo del Estado. En efecto, es la propia DGA el organismo sectorial con competencia ambiental en materia de aguas cuestión que solo puede hacer mediante el uso de herramientas más bien laterales, cosa que evidentemente dificulta su labor en este sentido, quedando por lo tanto a la deriva esta obligación del Estado.

\section{iv. El Dilema de Institucionalidad}

El referido dilema de institucionalidad es el problema de organización de las potestades del Estado en relación con el agua. Así visto, este no está en el mismo nivel que los anteriores sino que es transversal a ellos. El dilema de institucionalidad se observa como el área intermedia entre las instituciones fundantes de la regulación de aguas, sus principios y características y los demás dilemas antes descritos. Siendo así, es dable suponer que un cambio en la manera en que se configura este espacio intermedio aportaría en la solución de los problemas sin necesariamente implicar una modificación de las bases del sistema, aunque sí otorgándole cierta flexibilidad.

En lo que se refiere al dilema de institucionalidad, este trabajo sigue el concepto de Laughlin, quien señala que las estructuras organizacionales (institucionalidad) "son variables que intervienen entre los valores de alto nivel y los subsistemas tangibles, y son diseñados para guiar a estos últimos para expresar las perspectivas de los primeros” ${ }^{29}$. Así entonces, se entiende que la configuración institucional tiene entre sus consecuencias alimentar a los demás dilemas. Mientras lo ideológico normalmente está relacionado a valores fundamentales y por lo tanto de alto nivel, lo material y lo relativo al acceso son cuestiones más tangibles. Lo institucional en este escenario, funciona como mediador en los dilemas en sí mismo y también entre los distintos niveles.

El dilema de institucionalidad es consenso en la doctrina de aguas y la actual configuración es criticada por diversas razones por casi todos los autores. Así por ejemplo, Aran-

\footnotetext{
46 Valenzuela (2014) pp. 333-357.

47 Más aún, el autor es crítico de las otras reformas establecidas en 2005 y su relación con la protección del patrimonio ambiental. Así por ejemplo, a propósito de las patentes por no uso autor argumenta en el sentido de que forzar el uso del agua mediante la imposición de la patente podría haber provocado una mayor explotación del recurso con vistas a no perder el derecho o tener que pagar por él, cuestión que si bien podría haber tenido algún sentido en combatir la especulación con los derechos de aguas, claramente tiene un contrasentido cuando se le observa en el lente ambiental, pues tiende a la sobre explotación.

48 Boettiger (2014) p. 374.

49 Laughlin (1991) p. 212.
} 
da expresa "se debe estudiar e implementar una solución institucional que integre en una sola entidad los temas relativos a la gestión de la calidad del agua y los aspectos ambientales asociados al recurso hídrico..." ${ }^{50}$. Otro ejemplo es Vergara, quien también formula múltiples críticas a la institucionalidad, incluyendo la conflictividad entre los organismos que la componen, la falta de procedimientos internos, la falta de conocimientos adecuados y la falta de normas adecuadas, entre otras ${ }^{51}$.

Ese asunto es además reconocido ampliamente en el informe del Banco Mundial (BM) "Chile, estudio para el mejoramiento del marco institucional para la gestión del agua", de 2013, donde se señala como consenso en la materia que "La DGA necesita ser fortalecida en sus capacidades para que pueda llevar a cabo sus funciones en forma adecuada. En este sentido, tanto la insuficiencia de recursos como de autonomía, inciden en el débil presente de la DGA a nivel local"52.

Ahondando en los problemas institucionales, el BM reconoce (i) la falta de consolidación e integración de la información, (ii) la inadecuada delimitación y coordinación de funciones entre los organismos que intervienen en la gestión, (iii) la ausencia de una autoridad política superior, (iv) competencia presupuestaria entre los organismos, y (v) falta de coordinación de los actores responsables de la gestión a nivel local ${ }^{53}$. El informe antes referido expone cuáles son las alternativas de reforma para mejorar el marco institucional en Chile y propone como posibilidades el fortalecimiento de la DGA, la creación de una subsecretaría de recursos hídricos y la creación de una agencia nacional de aguas.

En su estudio, el BM hace un diagnóstico institucional utilizando como marco las brechas institucionales definidas por la $\mathrm{OCDE}^{54}$, el resultado de ese diagnóstico se presenta en la siguiente tabla.

TABLA 1

Diagnóstico de Brechas Institucionalidad de Aguas ${ }^{55}$

\begin{tabular}{|l|l|l|}
\hline BRECHA & ASPECTOS & DIAGNÓSTICO \\
\hline Administración & Ubicación, jerarquía, presencia & $\begin{array}{l}\text { DGA no promueve ni aprueba normas de } \\
\text { calidad y emisión ni planes de prevención y } \\
\text { descontaminación. }\end{array}$ \\
\hline Información & $\begin{array}{l}\text { Insuficiencia, calidad, dificultades } \\
\text { técnicas, etc. }\end{array}$ & Problemas en la generación y diseminación. \\
\hline Política & $\begin{array}{l}\text { Fragmentación de roles, falta de } \\
\text { apropiación política, falta de objeti- } \\
\text { vos claros, etc. }\end{array}$ & $\begin{array}{l}\text { Indefinición de Políticas y objetivos, falta } \\
\text { de coordinación interinstitucional, falta de } \\
\text { instrumentos participativos. }\end{array}$ \\
\hline
\end{tabular}

\footnotetext{
50 Aranda (2013) p. 119.

51 Vergara (2014).

52 Banco Mundial (2013b) p. 14.

53 Banco Mundial (2013b) pp. 15-16

54 Banco Mundial (2013b) p. 21. Lamentablemente el informe hace una referencia sin cita a la OCDE al senalar que las brechas institucionales fueron definidas por dicho organismo, sin que haya sido posible encontrar el documento al que se hace referencia.

55 Elaboración propia en base a BANCO Mundial (2013b).
} 


\begin{tabular}{|l|l|l|}
\hline Capacidad & Conocimientos técnicos & $\begin{array}{l}\text { Insuficientes recursos humanos en cantidad } \\
\text { y calidad. }\end{array}$ \\
\hline Financiamiento & Fuentes, gastos, etc. & $\begin{array}{l}\text { Insuficiente, variable y sin mecanismos } \\
\text { propios de recaudación }\end{array}$ \\
\hline Objetivos & $\begin{array}{l}\text { Claridad, lógica entre los objetivos } \\
\text { de distintas organizaciones, etc. }\end{array}$ & $\begin{array}{l}\text { Sin seguimiento de políticas ni de planifica- } \\
\text { ción estratégica, sin metas específicas. }\end{array}$ \\
\hline $\begin{array}{l}\text { Rendición de } \\
\text { cuentas }\end{array}$ & $\begin{array}{l}\text { Involucramiento de interesados, eva- } \\
\text { luación regulatoria, transparencia, } \\
\text { relación con gobierno. }\end{array}$ & $\begin{array}{l}\text { Criterios insuficientes y disparejos, fisca- } \\
\text { lización de políticas y planes bajo mando } \\
\text { único. }\end{array}$ \\
\hline
\end{tabular}

A pesar de que este dilema esté principalmente vinculado con los organismos públicos relacionados con el recurso hídrico, también tiene relación con las OUA y su funcionamiento, que como vimos es una de las claves del sistema regulatorio actual y de cualquier sistema racional de administración de un recurso como el agua y que por lo tanto cumplen una función pública.

Hearne y Donoso resumen las limitaciones de la institucionalidad chilena señalando que esta (i) ha fallado en crear arreglos institucionales que logren coordinar diferentes sectores económicos, (ii) carece de una agencia centralizada que genere políticas y coordine a los demás organismos, siendo que (ii) las funciones del Estado están muy fragmentadas y (iii) tienen competencias que se superponen y son poco claras, (iv) con poco presupuesto y (v) sin presión popular ${ }^{56}$.

Incluso autores que no se pronuncian a favor de un aumento de las potestades estatales reconocen la existencia de este dilema. Así por ejemplo Vergara, quien le llama "crisis institucional" en un trabajo reciente. El autor establece que existe una crisis del agua que se compone de cuatro crisis diversas; (a) Anomia administrativa, (b) Reconocimiento de la autogestión, (c) Comprensión de la libre transferibilidad, y (d) Ausencia de justicia especializada. Especificando, además, en la primera de ellas la imposibilidad de la DGA de cumplir con sus obligaciones ${ }^{57}$.

Con todo, existe acuerdo en que una cuestión transversal a los problemas en la regulación del agua es la orgánica en la que se produce y con la cual convive, cuya falta de armonía impide a los sujetos y a las normas desenvolverse adecuadamente. Una modificación institucional en ese sentido se hace inminente y ella debiera recoger la necesidad de tener un organismo con la experticia suficiente para abordar los otros dilemas antes descritos.

\section{CONCLUSIONES. LOS DILEMAS ENFRENTAN EL CAMBIO REGULATORIO}

Se han analizado los principales dilemas a los que se enfrenta la regulación de las aguas en Chile y el contexto en el que se realiza la presente investigación es un proceso de discusión sobre la necesidad de un cambio en los arreglos institucionales del agua, cuestión que tiene un lugar relevante en la agenda pública actual. Como es ampliamente reconocido

56 Hearne y Donoso (2014) p. 110.

57 Vergara (2014) pp. 15-17. 
en la literatura, este tipo de discusiones normalmente son el resultado de algún escándalo que fuerza una respuesta de las agencias públicas en relación con la regulación ${ }^{58}$.

Lo que se observa, es que ese escándalo probablemente es el hecho de que han existido algunos conflictos de notoriedad en relación con el agua. Es el caso particularmente de los conflictos en algunas localidades donde la crisis hídrica ha revestido mayor gravedad y ha provocado la movilización de la población. Asimismo, los medios de comunicaciones reportaron en variadas oportunidades la escasez de recursos hídricos, con énfasis en aquellas donde a la población rural se le abastecía de agua potable mediante camiones aljibe en diversas zonas del país, incluyendo zonas históricamente muy lluviosas ${ }^{59}$.

A propósito de ello es que el programa de la candidata presidencial que resultare electa en 2013, Michelle Bachelet, señala que la regulación actual ha generado "insuficiente resguardo del patrimonio ambiental, el deficitario acceso del mundo rural al agua y los nulos incentivos para gestionar la utilización de una fuente de agua común a partir de los derechos individuales, generando la sobreexplotación de múltiples acuíferos” ${ }^{100}$. Agrega además que la institucionalidad pública es insuficiente y que se avanzará hacia una normativa que tome en cuenta los fines sociales del agua como prioritarios, además de algunos otros temas específicos ${ }^{61}$.

Con ello se establece un vínculo lógico entre la existencia de un mercado de aguas y los problemas de sobreexplotación, falta de resguardo del patrimonio ambiental y problemas de acceso, de tal forma que todos estos quedan de alguna manera subsumidos en la existencia de este mercado, como problema principal. ¿Es eso real? ¿O es una impresión autorreferencial? De lo expuesto en este artículo se podría colegir que los problemas del agua son variados y que la existencia o no de un mercado, aunque pueda ser criticable, no es ni la causa ni la solución a ellos.

La lógica regulatoria impulsada entonces por el gobierno coincidiría con lo que Teubner ha llamado la autopoiesis de la ley, cuestión que produce una paradoja regulatoria y tiende por lo tanto a fallar en su objetivo ${ }^{62}$. Este concepto dice relación con la reproduc-

\footnotetext{
58 En este sentido por ejemplo, BLACK (2005). Reconoce que el detonante de los cambios o innovaciones en la regulación muchas veces es un shock externo, que si bien no podría explicar completamente la manera en que esa modificación se llevará a cabo, sí determina el comienzo de una discusión. En este artículo además, la autora hace referencia especialmente a este fenómeno de innovación regulatoria a propósito de regulación del riesgo, cuestión que también está presente en el caso pues la escasez de agua y los riesgos que ello conlleva son determinantes en generar la discusión respectiva.

59 Entre otras; http://salud.latercera.cl/noticia/nacional/2014/04/680-573639-9-sequia-26840-personasdependen-de-camiones-aljibe-para-consumo-de-agua-en-los.shtml]; http://salud.latercera.cl/noticia/ tendencias/2014/02/659-566452-9-lo-que-la-sequia-se-llevo.shtml; http://salud.latercera.cl/noticia/ nacional/2013/06/680-527511-9-sequia-en-la-iv-region-36-mil-personas-no-tienen-agua-y-camiones-deben. shtml; http://www.elmostrador.cl/kiosko/2013/12/13/2013-es-el-tercer-ano-mas-seco-desde-1866-y-crisishidrica-se-expande-al-sur/; http://www.eldinamo.cl/ambiente/2015/01/29/preocupante-zona-sur-atraviesapor-uno-de-los-verenos-mas-secos-de-los-ultimos-50-anos/; http:/www.pulso.cl/noticia/empresa-mercado/ empresa/2014/10/11-51048-9-delegado-del-agua-revela-diferencias-con-ciertos-parlamentarios.shtml

60 BaChelet (2014) p. 73

${ }^{61}$ Bachelet (2014) p. 73.

62 Teubner (2011) En el artículo el autor plantea una serie de mecanismos de autorreferencia de la ley que son catalogados como paradojas regulatorias y por lo tanto susceptibles de producir fallas regulatorias, vale decir
} 
ción intrínseca de un sistema, que en términos de la ley es la creencia de que la propia ley puede solucionar los problemas que supuestamente la misma ley ha creado, con escasa observación de los demás sistemas relacionados, incluyendo la realidad material. La autopoiesis y falta de conexión entre el supuesto diagnóstico y la innovación regulatoria propuesta es más observable en el proyecto de ley que finalmente fue presentado por el gobierno de la presidenta Bachelet en 2014, en el que se sigue una lógica similar, vinculando las sequías y los problemas de acceso al agua con una debilidad regulatoria que sería la falta de consideración del agua como bien nacional de uso público, lo que haría necesaria una modificación en ese sentido ${ }^{63}$. No se observa, sin embargo, cómo las innovaciones propuestas podrían modificar el supuesto del problema de sequías, siendo que solamente un porcentaje menor de las propuestas de modificación guardan relación con el problema de acceso, en especial aquellas que tienden a garantizar el derecho humano al agua y el saneamiento y a priorizar el uso en ese sentido.

Pero del análisis conjunto del proyecto original presentado en el Boletín 7543-12, modificado por la indicación sustitutiva antes dicha, el programa de gobierno y la literatura relevante, queda claro que nos encontramos frente a varios dilemas distintos, que son los que se expusieron en este trabajo. Resulta interesante destacar la longevidad de estos problemas. Ya en 1965, Steward hacía una clasificación similar a la que realiza este trabajo y distinguía entre los problemas de escasez absoluta de agua, los de escasez asociados a la reducción de la cantidad de agua disponible y los de escasez asociados al aumento de la demanda, sea por medios legales o de facto. A los primeros los asocia con los estudios sobre escasez física y al último como necesario de analizar dentro del contexto histórico-legal. Además, el autor reconoce una tensión que vendría desde el período posterior a la Edad Media en el Derecho Indiano y Español y que tendría que ver con dos corrientes doctrinarias, una que ve las aguas como cosas que pertenecen a los habitantes de una nación toda, y otra que cree que estas aguas pueden pasar por ley o en los hechos a ser parte del patrimonio privado de los individuos ${ }^{64}$. Esta tensión es prácticamente la misma que se denomina en este trabajo como dilema ideológico.

Este trabajo ha analizado las tres principales características del derecho de aguas enraizado en el Código de Aguas de 1981: la existencia de un mercado de derechos de aguas, la preponderancia de la gestión por parte de los usuarios y una escasa injerencia de la Administración del Estado. Luego, los dilemas detectados se clasificaron en 4 tipologías; ideológicos, material, de acceso y disponibilidad y de institucionalidad, cada una de las cuales fue explicada en su dimensión y ejemplificada en la literatura existente.

incapacidad de lograr el objetivo declarado por la regulación. En particular, cuando se refiere a la autopoiesis hace referencia a que el sistema legal se nutriría a sí mismo con independencia de otros sistemas sino solo con referencia a sí mismo.

63 Mensaje $\mathrm{N}^{\circ}$ 459-362, que formula indicación sustitutiva al proyecto de ley que reforma el código de aguas (Boletín N7543-12), de fecha 8 de septiembre de 2014.

64 STEWART (1970). 
La existencia de un mercado de aguas, que pareciera ser el foco del cambio regulatorio propuesto ${ }^{65}$, es solo uno de los arreglos institucionales problemáticos. Pareciera haber cierto consenso en que el mercado como sistema de repartición de la escasez ha fallado en dar solución a las diversas problemáticas que se forman en torno al agua y probablemente su eliminación o intervención es una cuestión necesaria. Sin embargo, ello no significará una mejora sustancial de los dilemas acá planteados, sino solo una variación, que aunque significativa, no es suficiente.

La discusión de nuevas regulaciones en relación con el agua debiera arrancar desde el reconocimiento y socialización de los diversos dilemas que enfrentamos y de la conciencia de que su solución probablemente no pasa por un mero cambio normativo, sino que requiere de la integración de soluciones desde diversas disciplinas, las que tienen que partir por un diagnóstico ordenado y consciente sobre los problemas que se enfrentan. El buen uso de la experticia al enfrentar este asunto es clave para lograr la construcción de instituciones justas, duraderas y eficaces.

\section{BIBLIOGRAFÍA CITADA}

Aranda Valenzuela, Pablo (2013) "Los Derechos de Aprovechamiento de Aguas en Chile y Su Marco Regulatorio", Revista de Derecho Escuela de Postgrado Universidad de Chile, No 4 diciembre 2013: p. 105-121.

Asociación de Empresas de Servicios Sanitarios AG (2013): Informe de gestión de la sequía 2014. Industria Sanitaria en Chile 2013. Disponible en http://www.andess.cl/descargas/noticias/201401-INFORME-GREMIAL-SEQUIA-ANDESS-ENERO-2014.pdf

BaCHelet, Michelle (2014): Programa de Gobierno 2014-2018, disponible en http://michellebachelet.cl/programa/, p. 73.

Banco Mundial (2013a): Estudio para el mejoramiento del marco institucional para la gestión del agua en Chile.

Banco Mundial (2013b): Chile, Estudio para el mejoramiento del marco institucional para la gestión del agua.

Banco Mundial (2011): Chile, Diagnóstico de la gestión de los recursos hídricos. Disponible en: http://www.dga.cl/eventos/Diagnostico\%20gestion\%20de\%20recursos\%20hidricos\%20en\%20Chile_Banco\%20Mundial.pdf p.8.

Bauer, Carl (1998): Against the Current: Privatization, Water markets and the State in Chile (New York, Springer).

Bauer, Carl (2014): “iLa Ley del Péndulo? Conflictos de Agua y Gobernanza en Chile desde 2005”, en: VII Jornadas de Derecho ambiental (Santiago, Thompson Reuters), pp. 637-670.

\footnotetext{
${ }^{65}$ Se señala que es el que pareciera ser el foco porque a pesar de que aparece en el discurso como el arreglo institucional que debe ser modificado, no se aprecia como las propuestas de nuevas regulaciones provocan esa modificación, sobre todo cuando aquellas que dicen relación con la perpetuidad y causa de los derechos de agua serían aplicables solo a derechos nuevos, cuestión de escasa relevancia en un escenario donde casi la totalidad del agua disponible (y buena parte del agua no disponible) ya ha sido asignada.
} 
BLACK, Julia (2005): "The development of risk based regulation in financial services: just “modelling trough"?”, en Black, Julia; Lodge, Martin y Thatcher, Mark (edit.) Regulatory Innovation (Northampton, Edward Elgar Publishing Limited): pp. 156-180.

Boettiger Phillips, Camila (2014): Variables Ambientales en el Código de Aguas. En VII Jornadas de Derecho ambiental, U. de Chile (Santiago, Thompson Reuters), p. 374.

Celume Byrne, Tatiana (2013): Régimen Público de las Aguas (Santiago, LegalPublishing).

Chile Sustentable (2010): Conflictos por el Agua en Chile: Entre los derechos humanos y las reglas del mercado. p. 47.

Chile Sustentable (2005): El agua en Chile, entre las reglas del mercado y los Derechos Humanos. Disponible en http://www.chilesustentable.net/wp-content/uploads/2011/07/Elagua-en-Chile-entre-las-reglas-del-mercado-y-los-derechos-humanos-Cartilla_Agua2.pdf

Dourojeanni, Axel y Jouravlev, Andrei (1999): El Código de Aguas de Chile, entre la ideología y la realidad, Disponible en: http://repositorio.cepal.org/bitstream/handle/11362/6380/S9910680_es.pdf?sequence=1 p. 10.

Espinoza Chacoff, Pía (2012): La regulación del servicio de agua potable y sus consecuencias en los indices de pobreza en Chile. Memoria para optar al grado de Licenciado en Ciencias Jurídicas y Sociales, Universidad de Chile (Santiago).

García, Aniza (2008): El Derecho Humano al Agua (Madrid, Editorial Trotta).

Heane, Robert y Donoso, Guillermo (2014): "Water Markets in Chile: Are they meeting needs?" en Easter, K. William y Huang, Qiuqiong "Water Markets for the $21^{\text {st }}$ Century" (New York, Springer) pp. 103-126.

IniCIaTIVA Agua QUe HAS DE Beber (2014). Agua en Chile: Diagnósticos territoriales y propuestas para enfrentar la crisis hidrica. Disponible en: http://www.aguaquehasdebeber.cl/wpcontent/uploads/2014/06/agua_chile_2014.pdf

Laughlin, Richard (1991): "Environmental Disturbances and Organizational Transitions and Transformations: Some Alternative Models” Organizational Studies, vol. $12 \mathrm{~N}^{\circ}$ 2: pp. 212-232.

LitTle, Christian y LaRA, Antonio (2010) "Restauración ecológica para aumentar la provisión de agua como un servicio ecosistémico en cuencas forestales del centro-sur de Chile" Revista Bosque, vol. 3 N 3: pp. 171-178.

Ochoa Tobar, Fernando (2011): "Algunas reflexiones en torno al derecho al agua, en especial sobre su recepción y ejecutabilidad en el ordenamiento jurídico Chileno", Revista Derecho y Humanidades, $\mathrm{N}^{\circ} 18$, Universidad de Chile: pp. 213-226.

ONU (2010): El derecho humano al agua y el saneamiento. Res. 64/292, Asamblea General.

Ostrom, Elionor (2012): El Gobierno de los Bienes Comunes (Ciudad de México, Fondo Económico de Cultura)

Prieto Andueza, Trinidad y Leyton Flórez, Patricio (2010): “Derechos de Aprovechamiento de Aguas: ¿'Titularidad de Papel?” en Derecho Ambiental en Tiempos de Reformas, Actas de las V Jornadas de Derecho Ambiental (Santiago, Abeledo Perrot) pp. 231-245.

Riquelme Salazar, Paulina (2014): "La regulación de los recursos hídricos como bienes nacionales de uso público, eventuales efectos de su incorporación en la Constitución Política de la República” en VII Jornadas de Derecho ambiental, (Santiago, Universidad de Chile-Thompson Reuters Santiago) pp. 277-304. 
Stewart, Daniel (1970): El Derecho de Aguas en Chile (Santiago, Editorial Jurídica de Chile).

Tapia valencia, Felipe (2014): “Tratamiento del Derecho Humano de Acceso al agua en el ordenamiento jurídico e institucional chileno", en VII Jornadas de Derecho ambiental, (Santiago, Universidad de Chile - Thompson Reuters Santiago) pp. 305-332.

Teubner, Gunther (2011): "And god Laughted... Indeterminacy, Self - Reference and Paradox in Law", German Law Journal, vol. 12 N 1: pp. 376-406.

Universidad de Santiago de Chile, Departamento de Gestión y Políticas Públicas, Facultad de Administración y Economía (2015): Estudio de Valores Sociales.

Valenzuela Jensen, Christian (2014): "Crítica a la ley 20.017 que modifica el Código de Aguas de 1981: aspectos en dirección contraria a la sustentabilidad”, en VII Jornadas de Derecho ambiental, (Santiago, Universidad de Chile-Thompson Reuters Santiago) pp. 333-357.

Vergara Blanco, Alejandro (1998): Derecho de Aguas (Santiago, Editorial Jurídica).

Vergara Blanco, Alejandro (2014): Crisis Institucional del Agua. (Santiago, LegalPublishing).

\section{NORMAS CITADAS}

Decreto con Fuerza de Ley No 458, de 13 de abril de 1976, Ley General de Urbanismo y Construcciones.

Decreto con Fuerza de Ley No 382, de 21 de junio de 2989, Ley General de Servicios Sanitarios.

Ley No 18.778 del 2 de febrero de 1989, Ley que establece subsidio al pago de consumo de agua potable y servicio de alcantarillado de aguas servidas. 